\title{
Acute myeloid leukemia in elderly patients: experience of a single center
}

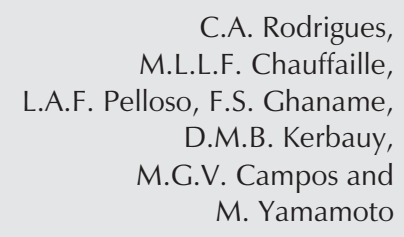

C.A. Rodrigues,

M.L.L.F. Chauffaille,

L.A.F. Pelloso, F.S. Ghaname,

D.M.B. Kerbauy,

M.G.V. Campos and

M. Yamamoto

Disciplina de Hematologia e Hemoterapia, Escola Paulista de Medicina, Universidade Federal de São Paulo, São Paulo, SP, Brasil

\section{Correspondence}

M.L.L.F. Chauffaille

Disciplina de Hematologia e

Hemoterapia, EPM, UNIFESP

R. Botucatu, 740, $3^{\circ}$ andar

04023-900 São Paulo, SP

Brasil

Fax: +55-11-5571-8806

E-mail: chauffaill@hemato.epm.br

Publication supported by FAPESP.

$\ldots \ldots \ldots \ldots \ldots \ldots$

Received August 20, 2002

Accepted March 19, 2003

\begin{abstract}
Acute myeloid leukemia (AML) is a disease predominantly of older adults. Treatment of AML in the elderly is complicated not only by comorbidities but also by the high prevalence of poor prognosis markers. Thirty-one consecutive unselected patients with AML older than 60 years (representing 33\% of all AML cases diagnosed at our institution during the same period) were followed over a period of 5 years (1997-2002). A high incidence of AML with multilineage dysplasia ( $45 \%$ ) and no favorable cytogenetic abnormalities but $62 \%$ intermediate and $38 \%$ unfavorable karyotypes were found. Sixteen patients $(52 \%)$ were selected for induction of intensive cytotoxic treatment and complete remission was achieved only by some of these intensively treated patients ( 7 of 16). Of these, 3 remained alive without disease (median: 11 months), 1 patient died shortly after complete remission, and 3 patients relapsed and died from refractory disease. Only 1 patient that was refractory to intensive cytotoxic treatment remained alive with disease under supportive care. Fifteen patients $(48 \%)$ were managed with palliative/supportive care: 7 received palliative treatment and supportive care, 8 received supportive care only, and 4 patients remained alive with disease under supportive care (median: 9 months). Mortality rate was $74 \%$ and overall survival at two years was $12 \%$. To the best of our knowledge, there is no previous report regarding elderly patients with AML in Brazilian subsets. The present data are similar to previously reported studies showing that elderly AML patients are not only older but also biologically distinct from younger AML patients, particularly in terms of the high incidence of poor prognostic karyotypes and resistance to therapy.
\end{abstract}

\section{Introduction}

Acute myeloid leukemia (AML) is predominantly a disease of older adults, with more than $50 \%$ of cases occurring over 60 years of age (1). AML in the elderly is asso-
Key words

- Acute myeloid leukemia

- Elderly patients

- G-banding karyotype

- Induction therapy

- Overall survival 
adults is limited by comorbidities that are frequent in this clinical setting (1). These features underlie the poorer outcome of these cases compared with younger patients (2).

However, prospective randomized studies clearly demonstrate that elderly patients benefit from more intensive induction therapy and particularly from full-dose application of anthracyclines and cytarabine $(2,3)$.

To the best of our knowledge, there are no previous descriptions of the clinical and cytogenetic features of AML in an elderly Brazilian population. Since Brazilian patients differ in ethnic background, endemic infectious diseases, socioeconomic aspects and medical support from North American or European patients, perhaps they need more specific approaches. Thus, we intended to evaluate the clinical and cytogenetic characteristics as well as the response to chemotherapy of an unselected group of Brazilian patients with AML older than 60 years admitted to a single institution over a period of 5 years $(1997-2002)$.

\section{Patients and Methods}

\section{Patients}

Ninety-two adult patients with AML were diagnosed at Hospital São Paulo (UNIFESP/ EPM) from January 1, 1997 to March 31, $2002,31(33 \%)$ of them being older than 60 years.

The AML diagnosis was defined in the presence of at least $20 \%$ blasts in bone marrow samples and patients were classified according to the new WHO classification (4). Being a retrospective study, no informed consent or approval of therapeutic measures was necessary by the Hospital Ethics Committee.

\section{Immunophenotype}

Immunophenotypic characterization of blast cells was performed using a panel of monoclonal antibodies with triple staining as previously described (5). Data acquisition and analysis were performed using a FACScalibur flow cytometer (Beckton Dickinson Immunocytometry Systems, San Jose, CA, USA) and the Cell quest software.

\section{Cytogenetics}

The karyotype was studied in bone marrow samples submitted to two short-term cultures with RPMI medium, 20\% fetal calf serum, $1 \% \mathrm{~L}$-glutamine and $1 \%$ antibiotics without mitogens (6). At least 15-20 Gbanded metaphases were analyzed and abnormalities were described according to ISCN (1995) recommendations (7).

\section{Treatment schedule}

Patients were treated according to their age, performance status by the Karnofsky index, and comorbidities. Patients younger than 75 years with a Karnofsky index $>75 \%$ and no major comorbidities (such as kidney or cardiac failure) were considered for intensive cytotoxic treatment. Patients who did not meet these criteria were considered for supportive/palliative care.

The intensive cytotoxic induction protocol consisted of cytarabine, $200 \mathrm{mg} / \mathrm{m}^{2}$ per day on days $1-7$, plus daunorubicin, $45 \mathrm{mg} /$ $\mathrm{m}^{2}$ per day on days $1-3$, or idarubicin, $12 \mathrm{mg} /$ $\mathrm{m}^{2}$ per day, on days 1-3. Complete remission was defined according to criteria reported by Cheson et al. (8), $<5 \%$ bone marrow blast cells and recovery of hematological parameters. When complete remission was not achieved, a second course of induction therapy was tried with mitoxantrone, $10 \mathrm{mg} / \mathrm{m}^{2}$ per day on days $1-5$, etoposide, $100 \mathrm{mg} / \mathrm{m}^{2}$ per day on days $1-5$, and cytarabine, 200 $\mathrm{mg} / \mathrm{m}^{2}$ per day on days $1-7$. After complete remission achievement, consolidation therapy was identical to induction therapy.

Palliative treatment consisted of monotherapy with hydroxyurea, 500-1500 mg/day, 
when white blood cell count $>20 \times 10^{9} / 1$, while supportive care was based on transfusional therapy, antibiotic prophylaxis or antibiotic therapy when needed.

\section{Statistical analysis}

The differences in treatment responses between the two groups, such as complete remission and survival, were evaluated by the Mann-Whitney test and the Kaplan-Meier method, with the level of significance set at $\mathrm{P}<0.05$.

\section{Results}

Thirty-one (33\%) patients older than 60 years were diagnosed during this 5-year period at Hospital São Paulo (UNIFESP/EPM). Age ranged from 61 to 85 years (median age $=70$ years) and there was a preponderance of females (1.6 female: 1 male). At diagnosis, median hemoglobin levels ranged from 4.1 to $11.7 \mathrm{~g} / \mathrm{dl}($ median $=6.7 \mathrm{~g} / \mathrm{dl})$ and white blood cell count from 0.9 to $112 \times 10^{9} / 1$ $\left(\right.$ median $\left.=8.6 \times 10^{9} / 1\right)$.

According to the WHO classification, 14 patients $(45 \%)$ had AML with multilineage dysplasia, 5 AML with maturation, 3 AML without maturation, 3 acute myelomonocytic leukemia, 2 monocytic leukemia, 1 monoblastic leukemia, 2 megakaryoblastic leukemia, and 1 AML developed after myelodysplastic syndrome therapy with an alkylating agent for vaginal lymphoma (Table 1).

A search for cytogenetic abnormalities was conducted in 29 patients and karyotype results were successful in 21 cases (72.4\%) (Table 1). No patient presented favorable cytogenetic abnormalities but intermediate or adverse prognosis karyotypes $(9,10)$. $\mathrm{Pa}$ tients with $\mathrm{t}(9 ; 22),-7, \mathrm{t}(3 ; 3)$, del(7q), and complex karyotype were described elsewhere (11).

We observed that adverse cytogenetic abnormalities were associated with a lower complete remission rate $(3$ of $6,50 \%$ ) as compared with patients with intermediate findings ( 3 of $4,75 \%$ ), although the difference was not statistically significant.

Sixteen patients were scheduled for intensive cytotoxic treatment, 7 patients received palliative treatment with supportive care, and 8 received supportive care only (Table 2).

\section{Intensively treated group}

Of 16 treated patients, 9 did not achieve complete remission. Of these, 8 died from refractory disease (after 0.5 to 6 months) and 1 refractory patient remained alive with disease under supportive care. Only 7 of 16

Table 1. Cytogenetic findings of 21 unselected elderly patients with acute myeloid leukemia and prognosis determined according to Grimwade et al. (10).

\begin{tabular}{lcl}
\hline $\begin{array}{l}\text { Prognosis group } \\
\text { stratification }\end{array}$ & $N$ & G-banding karyotype \\
\hline Favorable & & \\
Intermediate (62\%) & 13 & None \\
& & $48, X Y,+8,+22[14] / 46, X Y[1]$ \\
Unfavorable (38\%) & 8 & $47, X X,+11[5] / 46, X X[10]$ \\
& $46, X X$, add (21)(q22)[15] \\
& $45, X Y, t(3 ; ?)(q 26 ; ?),-7,-12,+$ mar[15] \\
& $46, X X, t(3 ; 3)(q 21 ; q 26)[20]$ \\
& $47, X X, \operatorname{del}(7)(q 31),+8[3] / 46, X X[5]$ \\
& $47, X X,+8[1] / 47, X X$, del(5)(q34), del(7)(q22), +8[4] \\
& $45, X Y,-7[16] / 46, X Y[4]$ \\
& $46, X X, t(9 ; 22)(q 34.1 ; q 11.2)[15]$
\end{tabular}

Note: Patients with $t(9 ; 22) ;-7, t(3 ; 3)$, del( $7 q)$, and complex karyotype were described elsewhere (11). The numbers within square brackets are the metaphase number analyzed as internationally standardized (7).

Table 2. Patient distribution according to type of therapy and clinical data.

\begin{tabular}{lccc}
\hline & Intensive cytotoxic & Supportive/palliative & Total \\
\hline $\mathrm{N}$ (patients) & 16 & 15 & 31 \\
Age (years) & $61-74(65)$ & $63-85(75)$ & $61-85(70)$ \\
Sex (female/male) & $9 / 7$ & $10 / 5$ & $19 / 12$ \\
Complete remission & $43 \%$ & $0 \%$ & $43 \%$ \\
Mortality & $12(75 \%)$ & $11(73 \%)$ & $23(74 \%)$ \\
Alive without disease & 3 & 0 & 3 \\
Alive with disease & 1 & 4 & 5
\end{tabular}


$(43 \%)$ intensively treated patients achieved complete remission, 6 with a first course of induction therapy and 1 after the second course (cytarabine, etoposide and mitoxantrone). Of these 7 patients, 1 died due to infectious complications shortly after complete remission achievement (less than one month) and 3 relapsed and died from refractory disease (median $=16$ months after complete remission). Only 3 patients remained alive without any clinical or hematological evidence of disease (continuous complete remission) for 26, 11 and 8 months, respectively (median $=11$ months). The mortality rate for this group was $75 \%$ (12 of 16 ) (Figure 1).

\section{Supportive treatment/palliative care}

Among 15 patients who underwent supportive treatment and/or palliative care, 11 died from infectious or bleeding complications. Four patients remained alive with disease under supportive care (median followup $=9$ months). The mortality rate for this group was $73 \%$ (11 of 15$)$.

\section{General mortality and survival}

Overall mortality rate was $74 \%$ and mean overall survival was 2 months $(0-50)$. Over-

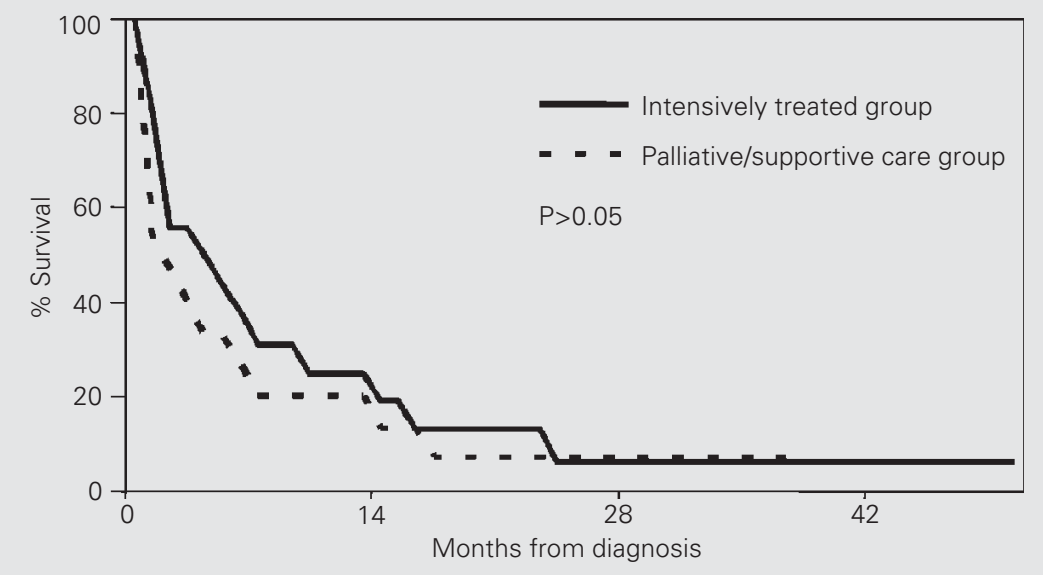

Figure 1. Overall survival of elderly Brazilian myeloid leukemia patients. The curves were constructed according to the Kaplan-Meier estimate. all survival was $22 \%$ at one year and $9 \%$ at two years. Mortality rate ( 75 vs $73 \%$ ), overall survival at one year (25 vs 20\%) and at two years (1 vs 6\%) did not differ between the intensively treated group and the palliative/supportive care group $(\mathrm{P}>0.05)$.

\section{Discussion}

The present study was undertaken to analyze different aspects of 31 consecutive elderly patients with AML. Some forms of AML that are associated with poor prognosis at any age are more prevalent in older adults (1).

We also found a high incidence of AML with multilineage dysplasia (45\%) and one case of AML developed after myelodysplastic syndrome therapy.

Therefore, no favorable cytogenetic abnormalities were found in the present study but, according to others, $62 \%$ of intermediate prognosis $(9,10)$ and a higher frequency $(38 \%)$ of unfavorable findings $(9,10)$. We observed that adverse cytogenetic abnormalities were associated with a lower complete remission rate (50\%) compared with patients with intermediate findings ( $75 \%$ ), although the difference was not statistically significant. Perhaps this difference could be detected in larger series.

Therapeutic results for patients older than 60 years admitted to clinical trials of intensive chemotherapy are largely unsatisfactory, with a median relapse-free survival usually lower than 12 months $(12,13)$. Currently, complete remission rates achieved with conventional chemotherapy range from 40 to $65 \%$ according to some investigators (13) and as shown here. In fact, complete remission was achieved only by some of the intensively treated patients (43\%). Further improvement of complete remission rate and duration will depend equally on the optimization of supportive care measures and the introduction of more effective therapeutic modalities (3).

The optimal management of AML in eld- 
erly patients remains a controversial issue. Complete remission rates after conventional induction chemotherapy progressively decrease after the age of 60 . This is explained by host-related factors and by differences in the biology of leukemia (13). The incidence of adverse prognostic factors (trilineage myelodysplasia, unfavorable karyotype, MDR1-positive immunophenotype) is higher in elderly patients $(1,13,14)$.

The two main strategies for improving outcomes in older adults with AML are to develop effective chemotherapeutic regimens with improved tolerability and to reduce drug resistance. But a satisfactory balance between efficacy and toxicity has not yet been achieved (1). Also, the use of growth factors to promote hematopoietic recovery has yet to yield consistent reductions in treatmentrelated morbidity or mortality (15).

Drug resistance can be modified by inhibiting drug efflux mechanisms (cyclosporine and valspodar) or by increasing sensitivity to cytotoxic agents, but these strategies have not yet been shown to significantly affect outcomes $(16,17)$.

Novel approaches including antibodytargeted chemotherapy (gentuzumab ozogamicin) may have the potential to improve prognosis for older adults with AML (18-20) but are still in the clinical trial stage.

Application of prognostic factors may permit to separate patients who would actually benefit from aggressive chemotherapy from those who should be offered attenuated/palliative treatments or enrolled in experimental trials of new drugs or biological/ immunological treatments (14).

Since $9.3 \%$ of the Brazilian population (21) living in São Paulo belong to the elderly segment and since life expectancy is 68.55 years in this region, it was interesting to find that $33 \%$ of adult patients with AML admitted to Hospital São Paulo were over 60 years of age, as compared to the $50-55 \%$ rate reported in other international series (1). This raises many questions such as whether the elderly do not have proper access to health care, or whether comorbidities or social aspects prevent the ideal diagnostic procedures, among other causes. Also, the female to male ratio in this age group in São Paulo is 1.17:1 (21) and in the elderly leukemia patients a 1.6:1 ratio was observed, an unexpected finding $(12,22)$.

The present results are similar to those reported in foreign studies, showing that this specific group of patients with AML is not only older but also biologically distinct. We could notice that even in a group of low socioeconomic profile in a developing country intensive treatment programs are applicable to the elderly with AML and that prolonged disease-free survival is possible for some, although it is clear that efforts are needed to pursue early diagnosis, prompt assistance and care, and to define specific groups tailored for new promising therapies.

\section{References}

1. Lowenberg B (2001). Managing therapy in older adult patients with acute myeloid leukemia. Seminars in Hematology, 38: 10-16.

2. Hiddemann W, Kern W, Schoch C, Fonatsch C, Heinecke A, Wormann B \& Buchner T (1999). Management of acute myeloid leukemia in elderly patients. Journal of Clinical Oncology, 17: 35693576.

3. Bassan R, Buelli M, Viero P, Minotti C \& Barbui T (1992). The management of acute myelogenous leukemia in the elderly: tenyear experience in 118 patients. Hematological Oncology, 10: 251260.

4. Jaffe ES, Harris NL, Stein H \& Vardiman JW (2001). World Health Organization Classifications of Tumours. Pathology and Genetics of
Tumours of Haematopoietic and Lymphoid Tissues. IARC Press, Lyon, France.

5. Bahia DM, Yamamoto M, Chauffaille ML, Kimura EY, Bordin JO, Filgueiras MA \& Kerbauy J (2001). Aberrant phenotypes in acute myeloid leukemia: a high frequency and clinical significance. Haematologica, 86: 801-806.

6. Chauffaille ML, Figueiredo MS, Beltrani R, Antunes SV, Yamamoto M \& Kerbauy J (2001). Acute promyelocytic leukemia: the study of $\mathrm{t}(15 ; 17)$ translocation by fluorescent in situ hybridization, reverse transcriptase-polymerase chain reaction and cytogenetic techniques. Brazilian Journal of Medical and Biological Research, 34: 735-743.

7. Mitelman F (1995). An International System for Human Cytogenet- 
ics Nomenclature (ISCN). Karger, Basel, Switzerland.

8. Cheson BD, Cassileth PA, Head DR et al. (1990). Report of the National Cancer Institute-sponsored workshop on definitions of diagnosis and response in acute myeloid leukemia. Journal of Clinical Oncology, 8: 813-819.

9. Grimwade D, Walker H, Oliver F et al. (1998). The importance of diagnostic cytogenetics on outcome in AML: analysis of 1,612 patients entered into the MRC AML 10 trial. The Medical Research Council Adult and Children's Leukaemia Working Parties. Blood, 92: 2322-2333.

10. Grimwade D, Walker H, Harrison G, Oliver F, Chatters S, Harrison CJ, Wheatley K, Burnett AK \& Goldstone AH (2001). The predictive value of hierarchical cytogenetic classification in older adults with acute myeloid leukemia (AML): analysis of 1065 patients entered into the United Kingdom Medical Research Council AML11 trial. Blood, 98: 1312-1320.

11. Pelloso LAF, Chauffaille MLL, Ghaname FS, Yamamoto M, Bahia DM \& Kerbauy J (2003). Cariótipo em leucemia mielóide aguda: importância e tipo de alteração em 30 pacientes ao diagnóstico. Revista da Associação Médica Brasileira (in press).

12. Lancet JE, Willman CL \& Bennett JM (2000). Acute myelogenous leukemia and aging. Clinical interactions. Hematology and Oncology Clinics of North America, 14: 251-267.

13. Pinto A, Zagonel V \& Ferrara F (2001). Acute myeloid leukemia in the elderly: biology and therapeutic strategies. Critical Reviews in Oncology/Hematology, 39: 275-287.

14. Harousseau JL (1998). Acute myeloid leukemia in the elderly. Blood
Reviews, 12: 145-153.

15. Bolam S \& Hamblin T (1999). Colony-stimulating factors in the treatment of older patients with acute myelogenous leukaemia. Drugs and Aging, 15: 451-460.

16. Ferrara F, Annunziata M, Copia C, Magrin S, Mele G \& Mirto S (1998). Therapeutic options and treatment results for patients over 75 years of age with acute myeloid leukemia. Haematologica, 83: 126-131.

17. Sonneveld P, Burnett A \& Vossebeld P (2000). Dose-finding study of valspodar (PSC 833) with daunorubicin and cytarabine to reverse multidrug resistance in elderly patients with previously untreated acute myeloid leukemia. Hematology Journal, 1: 411-421.

18. Goldstone AH, Burnett AK, Wheatley K, Smith AG, Hutchinson RM \& Clark RE (2001). Attempts to improve treatment outcomes in acute myeloid leukemia (AML) in older patients: the results of the United Kingdom Medical Research Council AML11 trial. Blood, 98: 1302-1311.

19. Estey EH (2000). How I treat older patients with AML. Blood, 96 : 1670-1673.

20. Bross PF, Beitz J, Chen G et al. (2001). Approval summary: gentuzumab ozogamicin in relapsed AML. Clinical Cancer Research, 7: 1490-1496.

21. IBGE, Censo Demográfico 2000 (2000). Malha Municipal Digital do Brasil 1997. [http://www.ibge.gov.br/cidadesat/xtras/temas.php].

22. McMullin M \& Mackenzie G (2001). Survival from AML in patient over 55 years of age in Northern Ireland: a discrete population. Hematology, 6: 103-110. 